\title{
Las mujeres en la revolución mexicana. Un punto de vista historiográfico
}

Ana Lau Jaiven

INSTITUTOO MORA

\begin{abstract}
Análisis de la historiografia sobre la participación de la mujer en la Revolución Mexicana. La autora divide los textos existentes en tres etapas y pone énfasis en la necesidad de proseguir y ahondar estos estudios utilizando la categoría analítica de género y considerando a la mujer como un sujeto histórico.
\end{abstract}

\section{$\mathrm{L}$} a participación de las mujeres en el movimiento revolucionario es para para aquellos(as) que estudian este proceso. Su interés se trasluce en el material publicado, tanto en nuestro país como en el extranjero. No obstante que apenas empezamos a conocer un poco más del tema, existen infinidad de preguntas aún no resueltas, aspectos, personajes y acontecimientos que permanecen a la espera de su historiador(a).

Los estudios publicados muestran sólo matices de la actuación social femenina y destacan, en lo general a las más conocidas, a las heroínas, a aquellas cuya huella es más fácil de rastrear, en tanto que cuestiones tales como las relaciones que se establecieron entre hombres y mujeres a raíz del conflicto, los cambios en los patrones de conducta en tiempos de guerra, los papeles asumidos por las mujeres y su respuesta frente a la sumisión de que fueron víctimas, todavía no ocupan el lugar que merecen dentro de los análisis del proceso revolucionario. ${ }^{1}$

$\mathrm{El}$ interés por consignar la participación femenina durante la revolución proviene no sólo de los hombres

1 Por ello pensé que sería interesante revisar parte del material bibliohemerográlico que existe sobre el tema. Soy consciente de que he dejado de lado mucho material, pero utilizo el que considero más conveniente para los fines del presente artículo. 
sino también de las mujeres; ambos se han interesado, aunque de distinta manera, por rescatar, analizar y explicar que la participación femenina no fue esporádica, secundaria ni estereotipada, sino que las mujeres como sujetos sociales deben ser consideradas dentro del contexto, en relación a sus demandas específicas y de acuerdo al bando o facción en que participaron.

En este sentido, el material bibliohemerógrafico que existe se puede dividir en tres grandes bloques: el primero, las fuentes primarias escritas durante el momento mismo de la oposición al porfiriato y en la guerra; el segundo, las obras publicadas entre los años de 1920 y 1950; y el tercero, las publicadas de 1950 a la fecha.

El primer periodo abarca aproximadamente un lapso de 1887 a 1917 y es el más interesante porque proviene de los mismos(as) participantes. El material conocido comprende publicaciones con características peculiares; las que pugnan por el mejoramiento moral de las mujeres, las que buscan la obtención de derechos e igualdad y por último las que influyen en la política en beneficio de ellas o bien las que se oponen a los cambios en su condición tradicional.

La cronología se inicia con la aparición del periódico dirigido por Laureana Wright de Kleinhans (18461896), Violetas del Anáhuac, ${ }^{2}$ en donde ya se plantea el problema del sufragio femenino, de la igualdad de

2 Violetas. Sobre Laureana Wright y cl periódico, véase Pasternac, "Periodismo" en Domenella y Pasternac (comps.), Voces, 1991, p. $399-448$. derechos para ambos sexos y se aboga por la instrucción de la mujer; el periodo concluye con la expedición de la Ley sobre Relaciones Familiares en abril de 1917, que intentó promover un cambio en las relaciones hombre/mujer dentro de la familia, en el matrimonio y con los hijos, después de que la revolución alteró usos y costumbres en algunas clases sociales, principalmente en la clase media así como en mujeres pertenecientes a estratos sociales más bajos.

En un primer momento, fueron las periodistas y las maestras -mujeres preparadas-las que dieron a conocer qué sucedía dentro del mundo femenino que a ellas les concernía; fueron las voceras de la corriente oposicionista y dejaron, expresadas en publicaciones periódicas, manifiestos, cartas y solicitudes, sus preocupaciones sociales que nos ayudan a conocer su trayectoria de lucha, sus ideas sobre la sociedad en que vivían y los cambios propuestos para un modelo de condición femenina. ${ }^{3}$

En los periódicos encontramos articulos que establecen el punto de partida para rastrear el pensamiento y acción femeninos; muestran la aspiración de un cambio significativo con relación a los patrones de conducta social y cultural imperantes hasta entonces y exhiben una manera de ser distinta, encaminada al ámbito privado, a replantear una relación de igualdad con los varones, y en el nivel político, a una mayor democratización.

3 Cartas, escritos y peticiones sc pucden encontrar en Lau y Ramos (Introd. y compilación), Mujeres, 1993. 


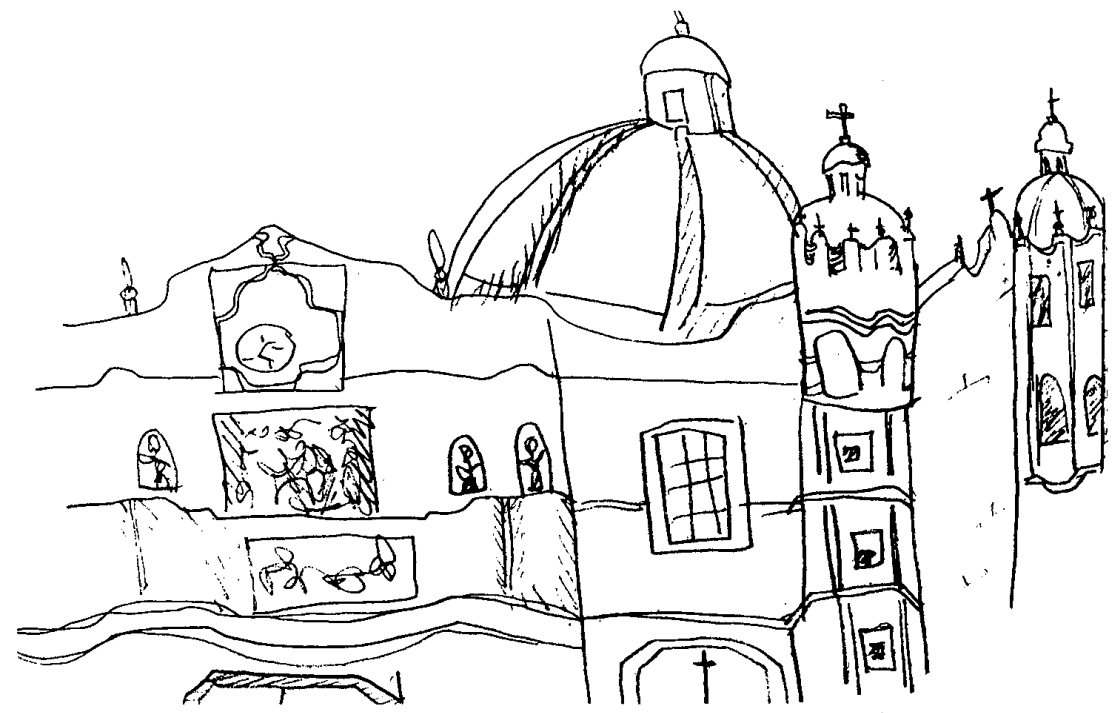

Periódicos como el ya mencionado Violetas del Anáhuac publicado entre 1887 y 1889 de Laureana Wright; El Recreo del Hogar, fundado en Mérida por Cristina Farfán; El Álbum de la Mujer, que encabezaba la feminista española Concepción Gimeno de Fláquer; La Mujer Mexicana, órgano de la sociedad feminista "Protectora de la Mujer"; El Hogar Mexicano de Laura Méndez de Cuenca, son una muestra de la actividad intelectual femenina que pugnaba por obtener beneficios para su género en lo relativo a la educación, al trabajo y a la vida doméstica y al mismo tiempo pretendía lograr el mejoramiento social de las mujeres de las clases menos favorecidas. Dichas publicaciones oscilaban entre la caridad y una actitud más polémica.
Además de los puntos de vista femeninos encontramos también las versiones masculinas que, difundidas en artículos y libros, manifiestan la preocupación sobre el impacto que podía alcanzar la proliferación de publicaciones femeninas y las demandas que éstas esgrimían. El objetivo de estos pensadores iba encaminado a aportar a la discusión elementos que contrarrestaran la rebeldía, que ellos denominaban como "feminista". En éstos se percibe una clara tendencia a aceptar la instrucción de la mujer con el único fin de que pueda realizarse con más ahínco en su maternidad y cuente con los elementos necesarios para desempeñarse como buena madre, ama de casa y esposa. 
Los positivistas ponían énfasis en que la vida privada era el único espacio permitido para la participación social de las mujeres y las excluían de un ámbito colectivo más amplio como era el de la política. Paradójicamente aceptaban que las mujeres de los estratos inferiores transgredieran estas normas y participaran como sujetos de explotación. Lo que los hacía caer en una doble moral que por un lado restringía a unas el acceso al mundo público, mientras que a otras las empujaba a participar por necesidades de sobrevivencia, sin que su papel se viera afectado. Como decía Andrés Ortega:

...porque para las mexicanas el pudor es su más rico tesoro, su aureola más brillante, su imán más atractivo; y como su contacto diario con los hombres, por causa de asuntos académicos, cientílicos y profesionales, tendría que teñir de carmín en más de una ocasión sus ruborosas mejillas, claro es que sería violentar su modo de ser, herirlas en mitad del corazón, asesinarlas proditoriamente, masculinizándolas con to que hoy torcidamente se llama feminismo. ${ }^{4}$

Hay que hacer notar que, mientras por una parte el pensamiento positivista proponía la sumisión de la mujer frente al hombre y prescribía que la mujer debía permanecer en el hogar y no participar en los asuntos públicos, y "en nombre de la diversidad biológica (éstos) pedian que se graduara su

\footnotetext{
4Ortega, "Feminismo", 1907, t. Il, p. 333, en Lau y Ramos, Mujeres, op. cit., pp. 9I-100.
}

educación para evitar la total emancipación de la mujer y que con ello desaparecieran las futuras madres $y$ esposas", 5 por la otra, las publicaciones femeninas exaltaban el papel de las mujeres en la educación, en el trabajo productivo e inclusive en el quehacer político.

En este sentido, el empleo femenino ${ }^{6}$ de finales del siglo XIX fue creando también las condiciones para una politización de las mujeres y su consecuente participación en organizaciones obreras, ${ }^{7}$ en grupos políticos y en los movimientos para derribar al gobierno porfirista. Su conciencia de explotación, tanto dentro del hogar como en el trabajo, creció rápidamente al grado que algunas mujeres no sólo participaron en el movimiento revolucionario sino en ocasiones también fueron vanguardia y líderes. ${ }^{8}$

La flagrante contradicción a la concepción positivista residía en el alto porcentaje de mujeres que se incorporaban a las labores productivas en fábricas y talleres, las que se empleaban

5 González, "Porfiriato", 1957, p. 577.

${ }^{6}$ En $1895,51.3 \%$ de la matricula educativa estaba compuesta por mujeres y para 1910, ésta ascendía a 61.4\%; en tanto que en el "ámbito laboral formal", en 1895 había 18.3293 mujeres emplcadas en actividades fabriles y en $1910 \mathrm{cl}$ número había ascendido a 199287 . Véase Vallens, Women, 1978.

7 las obreras participaron en asociaciones, mutualidades $y$ sindicatos desde antes de ta mitad del siglo XIX, agrupaciones que no sc integran en estos momentos; véase, por cjemplo, Mujer, crusmo, 1975 y Parcero, Condiciones, 1992.

8 Tal como lo consignan algunos Expedientes del Archivo Jlistórico de la Secretaría de la Delensa Nacional (AIISDN). 


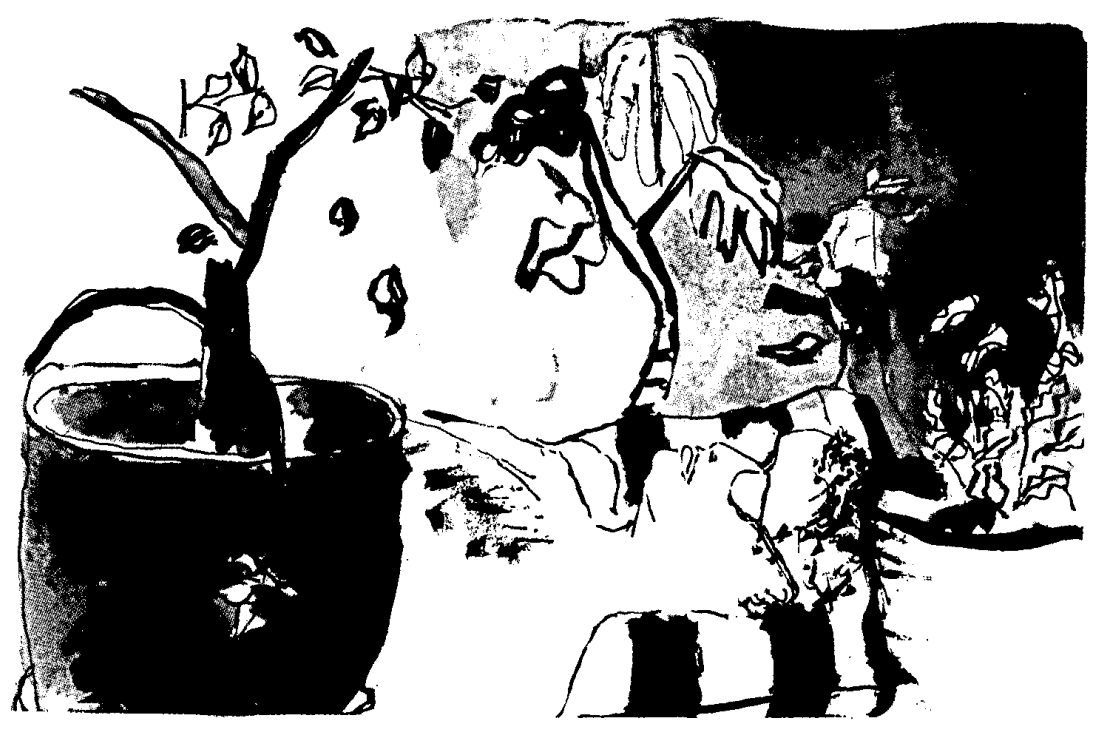

en trabajos informales como el servicio doméstico y la venta callejera de alimentos y otros productos, además de aquellas que participaban como periodistas, maestras, secretarias y burócratas y las que, desafiando la prescripción, llegaban a estudiar una carrera. (Vale citar los casos de las doctoras Matilde Montoya, Guadalupe Sánchez, Columba Rivera y Soledad Régules y de las abogadas Maria Asunción Sandoval de Zarco y Josefina Arce).?

Desde el ámbito cotidiano algunas mujeres subvirtieron su condición tradicional de amas de casa y abnegadas esposas para integrarse a la oposición 635.

9 Véase Gonzâlez, Historia, 1957 , pp. 634-

y militar dentro de grupos sindicales, mutualistas y revolucionarios. Apoyaron la causa antiporfirista con los medios que tenían a su alcance. Fueron madres, esposas, correligionarias, correos y espías. Y en los ámbitos y misiones en que participaron lo hicieron con igual intensidad y compromiso que sus compañeros varones.

Periódicos y semanarios como Vésper, ${ }^{10}$ dirigido por la periodista y militante antiporfirista, Juana Belén Gutiérrez de Mendoza (1875-1942), La Corregidora, de Sara Estela Ramírez (1881-1910), ${ }^{11}$ La Mujer Moderna ${ }^{12}$

10 Vésper, Justicia y I.ibertad, México, s.a.

11 Zamora, "Sara Estcla" 1980, pp. 163-169.

12 La Mujer Moderna, San Antonio, Texas, dicicmbre 1909 , t. I, núm. 1. 
de Andrea Villarreal y muchos otros aparecidos en la primera década de este siglo, son reflejo del empeño de estas mujeres por cambiar la inmutabilidad a que estaban sometidas a través de cambios políticos y por la instauración de una democracia que finalmente, pensaban, las haría acreedoras a derechos políticos.

Mujeres como Dolores Jiménez y Muro (1848-1925) quien militó en el magonismo, favoreció a Madero y por último se decidió por el zapatismo para combatir a Huerta; Juana Belén Gutiérrez de Mendoza (1875-1942), crítica acérrima del gobierno de Díaz, directora y fundadora de Vésper, tribuna desde la que atacó al régimen por lo que fue perseguida; como maderista fundó clubes de mujeres en apoyo al caudillo y solicitó el voto femenino. Elisa Acuña y Rossetti (1887-1946), compañera de lucha de Juana y periodista editora de varias publicaciones como Fiat Lux y La Guillotina; Andrea Villarreal (18811963), conocida magonista colaboradora de Regeneración, quien se desempeñó como correo y enlace de la causa entre México y Estados Unidos; en 1909 fundó el periódico mensual independiente La Mujer Moderna como órgano del club liberal Leona Vicario. Estas y muchas otras se entregaron a la causa con devoción y pelearon con la pluma por convicción. Sus artículos muestran una incipiente ideología de género ${ }^{13}$ que las

13 Utilizo el concepto tal y como lo mancja Tercsa de Lauretis en su artículo "La tccnología del género" en donde discute cómo se va constituyendo el género a partir de lenguajes y re- hacía combinar al mismo tiempo cuestiones políticas con innovaciones de indole privada en lo referente a la emancipación femenina.

Aquellas mujeres que se dedicaron al periodismo fueron activas promotoras de cambios en la situación para su género y también participaron en el terreno político en contra del gobierno de Porfirio Díaz; se comprometieron con los movimientos de oposición fundando organizaciones que protestaban contra los abusos del régimen y promovian la efectividad del sufragio y la no reelección, al mismo tiempo que pedían mejoras a la condición femenina. Al igual que algunos de sus compañeros sufrieron destierro, prisión y persecuciones. ${ }^{14}$

A partir de 1910 encontramos una creciente politización femenina; las discusiones en la prensa lo comprueban ya que las mujeres insertas en organizaciones que respaldaban a Madero se pronuncian y manifiestan en documentos donde combatían la reelección de Díaz o en cartas en donde solicitaban ayuda para sus hijos o fa-

presentacioncs culturales y cómo el géncro ticne la función de constituir a los inclividuos concretos en hombres y mujeres, por lo tanto jucga un papel importante en la construcción histórica ya que sigue realizándose en nucstros días como en el pasado. La jdeología de géncro es una posición en la existencia social en general, no inportando si las mujeres se perciben o no a si mismas como definidas por el géncro. Véasc lamos, Género, 1991, pp. 231-278.

14 Aurora Martíncz Vda, de Ilcrnández publicó una relación histórica en la que incluye los artículos publicados por su marido acerca de varias mujeres que participaron en la revolución, Véase Martínez, Antorchas, 1961. 
miliares; en estas últimas nos revelan cómo las mujeres a partir del ámbito privado se comunican con el poder. Las precursoras, en estos momentos, se definieron por uno u otro bando, pelearon pero también escribieron y las encontramos en los documentos como creadoras, firmantes o propagandistas de planes revolucionarios. ${ }^{15}$ Al lado de ellas, una pléyade de mujeres de todas las clases sociales se incorporó al movimiento en todas sus facetas: las localizamos como contrabandistas, correos, enfermeras, soldaderas, maestras, ...

También aquellas que si bien no participaron en el debate político, ni en la lucha armada, combatieron con la pluma por la superación de la mujer a partir del estudio, formaron clubes de ayuda a las desamparadas, lucharon por adquirir derechos y en ocasiones trabajaron por la protección de los desvalidos.

Temas como la violencia hacia las mujeres y la prostitución, esta última reglamentada en el porfiriato, no siempre fueron atendidos ya que.se consideraron "males sociales" producto de las circunstancias. Durante la lucha armada muchas mujeres tu. vieron que prostituirse para sobrevivir en tanto otras fueron violadas y sus hogares destruidos. Encontramos relatos que nos informan de la proliferación de "casas de mala nota", de burdeles en los pueblos por donde pasaban las tropas, en los cuales las mujeres se guarecian para obtener alimentos, seguridad y sobre todo, co-

15 Véase, Lau y Ramos, Mujcres, 1993, op. cit. mo una forma de encarar el abuso y las vejaciones. ${ }^{16}$

Por otro lado, al cambiar de lugar de residencia, de hombre o de bando, las experiencias sexuales de las mujeres se vieron alteradas, su concepción de entorno familiar se modificó y esta situación bastante generalizada también fue objeto de discusión y es posible rastrearla en los debates del Congreso Constituyente, donde el proyecto del artículo 22 proponía la pena de muerte al violador. ${ }^{17}$

Su papel tradicional de proveedoras se acentuó, durante la lucha armada, cuando se ocuparon de las tareas de la producción mientras sus hombres peleaban, sostuvieron sus hogares en pueblos y ciudades, así como también solucionaron las necesidades cotidianas de los soldados de ambos bandos, cocinaron, lavaron, parieron, fueron a la guerra y dispararon; al mismo tiempo otras actuaban como voceras de propuestas políticas.

Los documentos en que hemos investigado nos permiten conocer cómo participaron y qué pensaban sobre los asuntos políticos. Una de las principales representantes del feminismo en la Revolución fue Hermila Galindo ${ }^{18}$ (1885-1954), quien además de publicar el semanario La Mujer

16 Véasc Rocha, "Nuestras" en llistorias, 1990-1991, núm. 25, pp. 120-121; Urquizo, Recuerdo, 1985.

${ }^{17}$ Diario de los Dehates del Congreso Consliluyenle, 1916-1917, Imprenta del Gobicrno, 1917, 1. 11.

18 Gabricla Cano ha examinado las propuestas feministas de liermila Gallindo, véase "Revolución", en Duby y Perrot (coords.), IIistoria, 1993 , t. 5 , pp. $685-695$. 
Moderna, ${ }^{19}$ funcionó como secretaria de Venustiano Carranza y fue ardiente defensora de su doctrina que propagó con entusiasmo en escritos y discursos.

Durante el periodo armado de la revolución se desarrolló una nueva forma de relaciones entre hombres y mujeres; ${ }^{20}$ éstas se desenvolvieron en todos los ámbitos y las feministas se apresuraron a publicitar estos cambios.

Tal fue el caso, por ejemplo, del primer editorial de La mujer moderna, aparecido el 16 de septiembre de 1915, en que Hermila Galindo escribía, refiriéndose al aniversario de la Independencia:

Deseamos honrar este aniversario de redención, inaugurando nuestras tareas periodísticas, con las que deseamos coadyuvar a la redención de la patria, la redención de los principios salvadores y la redención de la mujer, levantando el espíritu femenino a ta altura de su deber y su derecho, pari que no permanezca por más tiempo impasible ante la solución de los más trascendentales problemas sociales y políticos, que afectan tanto al hombre como a la mujer, que es su compañeral y su igual. ${ }^{21}$

19 Suponemos que toma su nombre como homenaje al periódico de Andrea Villarreal cuyo primer número apareció en diciembre de 1909.

20 Ya fuera porque perdian a su pareja y sc arrimaban a otro hombre. O bicn, otra forma dc intercambio de parcja erin los raptos. Fen la clase media se dicron muchas separaciones por ausencia del cónyuge, quien se quedaba a vivir en alguna región distante.

21 “I Iaboremos!” La mujer Modema Senanario iluslrado, año 1 , núm. 1 septicmbre 16, 1915.
La actuación femenina fue tan visible que los líderes se vieron obligados a dirigirse a las mujeres prometiéndoles igualdad de derechos y privilegios.

La mayoría de los pasatiempos literarios y de los elogios tributados a la revolución dedican alguno que otro párrafo al papel de las mujeres mexicanas en la sociedad, empleando con frecuencia esos clogios para subrayar los alcgatos en pro de la plena intcgración de la mujer en la nación mexicana. ${ }^{22}$

Tales fueron los casos de los proyectos, en el nivel legislativo, dentro de las distintas facciones de la revolución. La exigencia de hacer coincidir la realidad con los cambios en las relaciones personales y de ¿género? que la guerra había alterado hizo que se argumentara la necesidad de reformar disposiciones existentes. Discusiones $y$ alegatos se entablaron durante la reuniones de la Soberana Convención de Aguascalientes ${ }^{23}$ para otorgar protección a las mujeres. Entre los zapatistas, por ejemplo, en 1915, se emitió un proyecto de ley sobre el matrimonio $^{24}$ en el que se aceptaba el divorcio y se borraba el estigma de ilegitimidad para los hijos. Los constitucionalistas por su parte, promulgaron en 1915 una ley del divorcio 25 y en 1917 la Ley de relaciones familiares. ${ }^{26}$ Esta legislación manifiesta la preocupación que los revolucionarios mostraban por co-

22 Turncr, Dinámica, 1971, p. 245.

23 Barrera, Cronicas, 1965.

24 "Proyecto de ley sobre el matrimonio" en Rocha, Album, 1991, vol. iv, p. 268.

${ }^{25}$ Lau y Ramos, Mujeres, 1993, pp. $311-326$.

26 En ibid., pp. 327-349. 
rresponder con algunos derechos a la participación de las mujeres y al mismo tiempo llevar a cabo cambios que ayudaran a la modernización del país. Sin embargo, prejuicios milenarios entre los que la religión jugaba un papel muy importante, harian que la obtención del sufragio tuviera que esperar todavía casi cuatro décadas.

El hecho de que las mujeres fueran consideradas como una fuerza política capaz de ser atraída por las propuestas revolucionarias, propició la organización de dos congresos feministas a los que asistieron una gran mayoría de maestras, en Mérida, Yucatán, en enero y noviembre de 1916. El objetivo de ambas reuniones giró en torno a la discusión y examen del grado de participación de las mujeres en la nueva sociedad que se pretendía establecer. Por la lectura de los estatutos, de los trabajos y los debates realizados durante las sesiones, sabemos qué temas preocupaban al gobierno constitucionalista y a las asistentes; ${ }^{27}$ los argumentos coincidieron en admitir la importancia de reconocer la capacidad de las mujeres, fomentar su instrucción y solicitar que se modificara la legislación a fin de que pudieran participar de manera más activa en la sociedad.

No obstante las peticiones y la participación organizada de muchas mujeres, los constituyentes no consideraron que el sufragio era conveniente para ellas, aduciendo que si bien había algunas mujeres excepcionales capa-

271916 El Primer Congreso Feminisla de Yucatán. Anales de esa memorable Asamblea, 1975. citadas para participar en la vida pública, la gran mayoría no lo estaba. Y añadian:

La diferencia de los sexos determina la diferencia en la aplicación de las actividades; en el estado en que se encuentra nuestra sociedad, la actividad de la mujer no ha salido del círculo del hogar doméstico, ni sus intereses se han desvinculado de los de miembros masculinos de la familia; $[\ldots]$ las mujeres no sienten, pues, la necesidad de participar en los asuntos públicos, como lo demuestra la falta de todo movimicnto colectivo en ese sentido. ${ }^{28}$

El razonamiento esgrimido por los constituyentes, eludió la participación femenina durante el periodo de lucha y su explicación manifestó el temor de que esas mujeres participaran políticamente como ciudadanas iguales. Con este argumento dejaron claro que soslayaban la participación femenina en la guerra ya que consideraban que hombres y mujeres tienen papeles sociales diferentes y que reforzar las labores domésticas era el único ámbito permitido para ellas.

La obtención del voto entrañaba el reconocimiento de las mujeres como sujetos políticos con capacidad para la conducción y acceso a la toma de decisiones dentro de la sociedad. La negación del voto en sí, implicó que se excluía a las mujeres no sólo de la posibilidad de participar políticamente dentro del proyecto por el que algunas habian luchado y al que habían defendido, sino que se las hacía a un

28 Diario de los Debates del congreso Constiluyente, op. cit.; Bremaunt\%, Sufragio, 1937. 


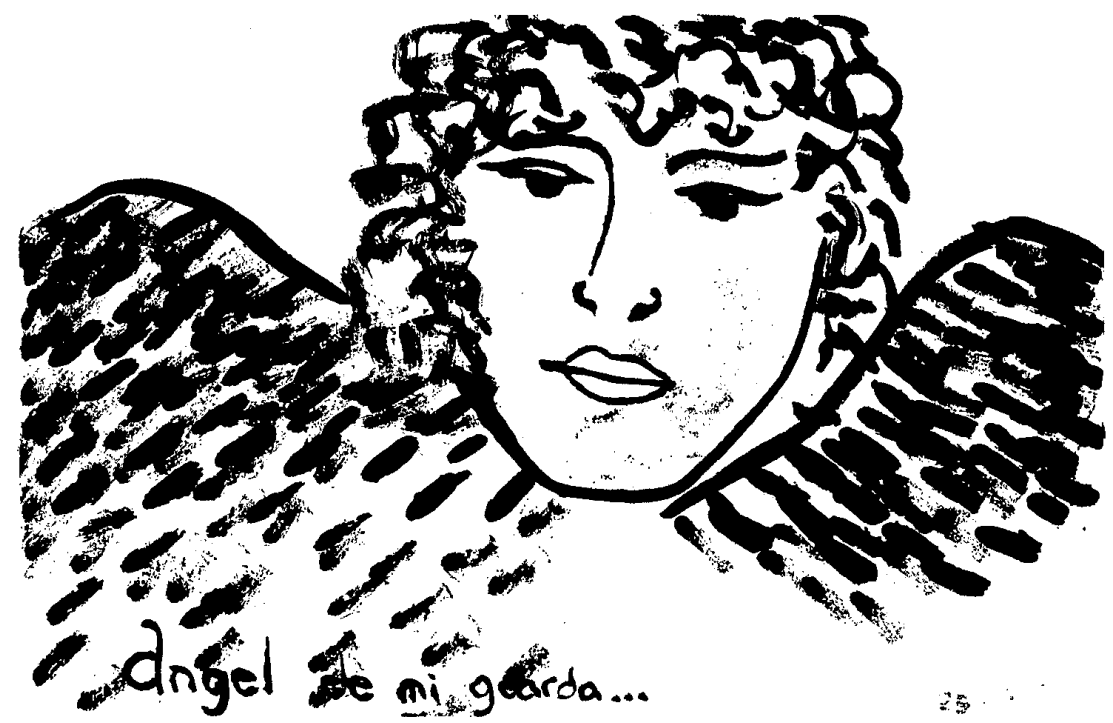

lado en las decisiones acerca de la conducción de la sociedad que se estaba conformando.

Pero las mujeres sufragistas no quitaron el dedo del renglón.

Terminada la lucha armada, encontramos una generación de escritoras que en algunos casos habían participado en la revolución o que por su edad, vivieron el movimiento tangencialmente. Sus escritos, con afanes políticos, con miras a la obtención del sufragio, rescataron la participación de sus congéneres y fundamentaron su actividad política a partir de la gesta armada. Nuevas leyes, una apertura mayor de puestos de trabajo y de educación para las mujeres permitió su incorporación en la vida económica del país. Así encontramos entre 1920

y 1950 una serie de publicaciones que inician el rescate de la actuación femenina durante la revolución. ${ }^{29}$

Estas mujeres en su momento, se apoyaron en sus antecesoras para crear el vínculo que justificara sus peticiones, sus motivaciones politicas y les permitiera organizarse con miras a la obtención de derechos sociales; también dejaron constancia por escrito de sus ideas. Cabe mencionar aquí sólo algunas publicaciones que parten en un primer momento de una des-

29 Las reseñas de los títulos clegidos a continuación se restringen a aquellas obras que tratan del desempeño de las mujerces durante ha revolución. Estoy consciente de que existen muchas más publicaciones pero el análisis sc reduce sólo a las que reivindican a las mujeres que lucharon durante el periodo revolucionario. 
cripción detallada de los nombres y de la reivindicación de las acciones de aquellas que participaron en la revolución.

A través de la lectura de libros como el de Margarita Robles de Mendoza, La evolución de la mujer en México (1931); de María Ríos Cárdenas, La mujer mexicana es ciudadana. Historia con fisonomía de una novela de costumbres (1940); de Ana María Hernández, La mujer mexicana en la industria textil (1940), nos permiten analizar a la revolución desde la perspectiva del tiempo y la distancia. A través de ellos conocemos las premisas que planteaban y que giraban en torno a lo que se debatía dentro del país: incrementar la educación del pueblo como panacea para erradicar los males que lo aquejaban y hacer entrar a México en una etapa de progreso.

Margarita Robles de Mendoza, maestra, socióloga y feminista, delegada de México en la Comisión interamericana femenina de Washington, aducia refiriéndose al voto femenino que

Para que las leycs dejen de ostentar ese sórdido matiz egoísta y unilateral que los hombres les han dado desde tiempos inmemoriales, y para que las nuevas leyes igualitarias se incrusten defìnitivamente en las costumbres, es necesario que la mujer tome parte directa en la cdificación de las construcciones legislativas, y en su antecedentc indispensable de acción social y de actividad política. ${ }^{30}$

30 Robles de Mendoza, "Emancipación", $I t$ Universal, 20 de diciembre de 1929 en Evolucion, s.e., 1931, p. 66.
Pero la necesidad de que las mujeres cumplieran con sus deberes la llevó a plantear que: para darles derechos había que educarlas primero y para ello proponía la creación de escuelas que las preparasen para enfrentar los retos que el país reclamaba.

En el mismo sentido, María Ríos Cárdenas, presidenta de la Confederación Femenil Mexicana, dedica una parte de su libro a la mujer en la guerra y se apoya en su participación "desde las soldaderas hasta las que han ostentado un grado militar, (ya que) han contribuido a crear y sostener la soberanía de la República mexicana",31 todo ello a fin de respaldar sus propuestas para obtener el sufragio durante la administración cardenista.

Otro ejemplo es el de una conferencia dictada en septiembre de 1937 por Matilde Rodríguez Cabo, "La mujer y la revolución" en que la autora, imbuida por el pensamiento socialista de los treinta en México, cuando se discutía la posibilidad de que se otorgara el sufragio femenino (que Cárdenas prometió y no cumplió), se valió de la actuación femenina revolucionaria para argumentar que "un gobierno que quiere solucionar los problemas del pais tiene que considerar dentro de su programa el capítulo relativo a la mujer". ${ }^{32}$ Rodríguez Cabo revisa los conceptos emitidos por los hombres para concluir que ellos sólo habían observado a la mujer desde el punto de vista sentimental, olvidando considerar el aspecto de la mujer trabajado-

31 Ríos, Mujer, 1942, p. 130.

32 Rodrígucz, "Mujer", Conferencia dictada en cl frente Socialista de Abogados, 1937, p. 14. 
ra, productora, educadora y factor determinante en la organización social.

No obstante, en momentos como éste de efervescencia política, la autora anotaba

corresponde a nosotras las mujeres, en primer lugar, y en cumplimiento de un elemental deber de justicia, recordar y conceder el lugar de iniciadoras a aquellas valerosas mujeres que nos precedieron en la lucha y nos marcaron el derrotero a seguir.....33

A pesar de la valerosa actuación tanto en el frente de batalla como desde la intimidad del hogar, considera que la actividad de las mujeres no ha sido tomada en cuenta, y espera que con la obtención de derechos civiles, "la revolución efectivamente empiece a saldar la cuenta que tiene con la mujer". 34

En 1940 se publicó La mujer mexicana en la industria textil, escrito por la inspectora federal del Trabajo, Ana María Hernández quien elaboró un estudio del trabajo textil de la mujer mexicana a través del tiempo y destacó a las mujeres activas en el movimiento obrero durante la revolución. Mientras que Fortino Ibarra de Anda, dedicó un volumen entero de su libro sobre el periodismo a estudiar a Las mexicanas en el periodismo (1935). 35

33 Ibid., p. 15.

${ }^{34}$ Ibtal, p. 30.

35 Hernándè, Mujer, 1940; lbarra, Periodismo, 1937. ( $\Lambda$ parcece también con Imprenta Mundial, 1935). De ambos libros se han extraido datos para muchos artículos que se refieren a las mujeres en la revolución, un cjemplo:
Para finalizar con esta etapa, en 1942 se publicó un artículo académico de Lillian Estelle Fisher, ${ }^{36}$ en donde la autora subraya que las mujeres luego de la revolución, obtuvieron más derechos de los que tenían antes, no obstante en el momento en que esto se escribe, su posición ${ }_{2}$ continuaba siendo inferior a la del hombre. También resalta, como uno de los principales logros obtenidos con la revolución, la ley del divorcio y la protección de las mujeres trabajadoras que otorga el artículo 123 , concluye afirmando:

Las mujeres están trabajando para resolver sus propios problemas e interesándose en el mejoramiento de la educación de los pobres, servicios comunitarios y muchas otras cuestiones vitales que alectan a su país. Cuando obtengan el voto en toda la república, que por ahora es su meta principal y para la cual sólo es cuestión de tiempo, obtendrán el bienestar para su sexo y se convertirín en el fictor decisivo del destino humano. ${ }^{37}$

El último bloque de estudios relativos a la participación femenina en la revolución, abarca de la década de los cincuenta hasta el día de hoy. En éste distinguimos dos tipos de obras: las escritas para exaltar la labor revolucionaria de las mujeres con motivo del otorgamiento del voto en 1953 y los estudios más académicos que buscan

Filosofia y Letras. Revista de la Facullad de Filosofía, México, enero-dicicmbre de 1956, t. $\mathrm{xxx}$, núms. 60-61-62-

36 Fisher, "Inlluence" en IIis/panic, vol. XXII, núm. 1, pp. 211-228.

37 Ibid., p. 228. 
explicar las causas de la incorporación femenina a la lucha armada, exponer cómo fue esa actuación y elaborar un conjunto de hipótesis que permitan analizar el papel de las mujeres en la guerra y las relaciones que éstas establecieron con sus contrapartes masculinos.

Recurriendo a una tónica glorificadora y triunfalista de la actuación de las mujeres en la revolución, encontramos a la periodista veracruzana Artemisa Sáenz Royo, "Xóchitl", quien tras la obtención del sufragio en 1953 , dedicó su libro al presidente Adolfo Ruiz Cortines impulsada

por la gran oportunidad de dar a conocer algunos hechos ignorados de varias mujeres que lucharon en favor de la ideología revolucionaria, y por los caídos, por los opresos por un régimen autócrata e injusto. ${ }^{38}$

Sáenz Royo enlista en la Historia político social cultural del movimiento femenino en México, 19141950 , las acciones en que las mujeres resaltaron. Años después, en el cincuentenario de la revolución, añade más nombres al panteón de las heroínas en Semblanza: mujeres mexicanas, revolucionarias y guerreras, revolucionarias ideológicas. ${ }^{39}$ Tiene un mismo sentido reinvindicatorio y de rescate, el libro de Aurora Martínez Vda. de Hernández, Antorchas de la revolución. ${ }^{40}$

38 Sáenz, Historia, 1954. p. 9.

39 Sáenz, Semblanza, 1960.

40 Aurora Martíncz, op. cti.
El primer intento serio por examinar a la mujer en la revolución es el libro La mujer en la revolución mexicana ${ }^{41}$ de Angeles Mendieta de Alatorre, quien tiene el mérito de haber consultado los expedientes del Archivo Histórico de la Secretaría de la Defensa donde se consignan los nombres de las mujeres que participaron activamente en la revolución, y aprovechó además, las semblanzas aparecidas en El Nacional ${ }^{12}$ que consignaban las hazañas de las revolucionarias.

El libro aporta una visión general de la participación política de las mujeres mexicanas durante el periodo 1900-1953, concediendo especial atención a la etapa revolucionaria así como a las biografias de las más sobresalientes.

El interés de esta investigación reside en que el tema se abordó con mayor seriedad y por lo tanto despertó, a partir de las referencias bibliohemerográficas que aporta, la curiosidad de otros especialistas para continuar con investigaciones que permitan extraer en última instancia, una visión integral de la actuación de las mujeres, que evite o margine los estereotipos aún vigentes en gran parte de la sociedad. De la misma Angeles Mendieta hay publicadas dos biografias, una de Juana Belén Gutiérrez de Mendoza y otra de Carmen Serdán. ${ }^{43}$

Las investigaciones posteriores al libro de Mendieta aportan material in-

41 Mendicta, Mujer, 1961.

42 La mayor parte de éstas fucron escritas por Rubén García en 1959.

${ }^{43}$ Mendieta, Carmen Serdán, 1971 ; Juana B. Gutiérrez de Mendoza, 1983. 
teresante acerca de acontecimientos en los que las mujeres fueron las protagonistas principales, también analizan sus objetivos políticos y en ocasiones hasta sus motivaciones. Ya no son sólo heroínas sino mujeres de carne y hueso con pasiones y debilidades humanas, observadas dentro del contexto histórico en que les tocó vivir.

Entre estas obras encontramos las que están escritas en inglés y manifiestan la fascinación que la revolución ha ejercido entre la comunidad académica del vecino país y que, unida al renacimiento del movimiento feminista norteamericano, buscó las raíces de su lucha en el pasado, lo que permite entender la proliferación de estudios que ahora comentamos. Mencionaremos sólo algunos: en 1967 apareció el artículo de Frederick C. Turner ${ }^{41}$ que disparó la nueva corriente académica de estudios referidos a las mujeres en la revolución. Su tesis parte de examinar el grado de nacionalismo que impidió a las mujeres mexicanas adquirir el sentido de pertenencia, como miembros activos de la comunidad nacional y en cambio dice que las mujeres mexicanas hubieron de buscar ese complemento en las lealtades de inclole religiosa. ${ }^{45}$ Además examinó los cambios que la guerra provocó a nivel tecnológico, familiar y social y, advirtió que la revolución ha sido considerada como la "madre de los derechos políticos de las mujeres y de su emancipación so-

\footnotetext{
4h Turncr, "Iffectos", en /listeria, vol. 16, núm. 4, abril-junio de 1967.

45 Turner, Dinámica, 1971, pp. 238-239.
}

cial" por lo que la creación del mito de las heroínas resultó natural.

Shirlene Ann Soto, 46 al igual que Turner considera que la religión fue el obstáculo que impidió la plena integración de las mujeres a la vida nacional luego de la revolución. El objetivo de su investigación gira alrededor del rescate de las mujeres ignoradas por la historia y pretende incrementar el conocimiento de las dinámicas que se crearon durante la revolución mexicana e informar a los lectores norteamericanos acerca de las mujeres que participaron. Su estudio nos introduce en el contexto revolucionario en que destaca la actuación femenina a partir del lugar que las mujeres ocupaban en la escala social y del bando o facción al que se afiliaron. El énfasis de su estudio reside en analizar con mayor profundidad el movimiento de mujeres en Yucatán desde los congresos feministas auspiciados por Salvador Alvarado hasta las reformas promovidas por Felipe Carrillo Puerto durante su gobierno entre 1922-1924. El problema que presenta y que es reflejo del momento en que fue escrito es que habla de las mujeres sin considerar sus relaciones con los otros grupos sociales, es decir, todavía reivindica la actuación femenina per se, sin preocuparse por examinar su función social y las vinculaciones que se establecieron entre hombres y mujeres.

Por último, el libro de Anna Macías, ${ }^{17}$ forma parte con los dos anterio-

\footnotetext{
46 Soto, Woman, 1969 y también cl artículo: "Yucit:an's", 1985, 12 p.

17 Macias, Agcinst, 1982. Las ponencias previas que la llevaron a cscribir el libro son: "Re-
} 
res, de la misma corriente de rescate del feminismo y pretende demostrar la existencia de un movimiento feminista en nuestro pais entre 1890 y 1940. El aporte de esta obra reside en la vastedad bibliohemerográfica, en la información sobre la historia de las demandas feministas y en haber esbozado las diferencias de género entre hombres y mujeres; características que hoy ocupan un lugar principal en los análisis de las académicas.

La necesidad de evaluar el comportamiento femenino se hizo patente en estos años; era necesario rescatar a las mujeres como sujetos sociales para justificar los avances y compartir una memoria colectiva en relación al grupo social específico. En 1975, el Año Internacional de la Mujer favoreció la aparición de algunos artículos de rescate como el de María Antonieta Rascón, Marcela Lagarde y Susana Vidales. 18

Debe señalarse que las historiadoras mexicanas también nos hemos preocupado por estudiar a nuestro género, y aunque los libros son escasos, creo que significan un avance y una nueva manera de abordar e interpretar la historiografia de la revolución. ${ }^{49}$

volution" en Hankc (comp.), Iatin America, Boston, 1974; "Woman" en The Americas, julio de 1980, vol. 37, núm. 1, pp. 53-82. Y cl artículo "Carrillo Puerto" en Lavrin (comp.), Mujeres, 1985, pp. 329-316.

48 Rascón, "Mujcr" 1975, pp. 139-17/4; lagarde, "Memoria", septicmbre de 1980; Susana Vidalcs, "Madres", 1980.

49 Carmen Ramos y $\Lambda$ sunción Lavrin claboraron un análisis historiográlico de las investigaciones que se han abocado al estudio de las
En este sentido se puede constatar el interés por conocer la historia de las mujeres que obedece, en buena medida, a la preocupación por situarlas como sujetos históricos, subrayando su presencia, su importancia y significado en una sociedad y en un momento determinado; para ello la categoría analítica de género ha resultado de gran utilidad porque permite examinar la forma en que las sociedades organizan las relaciones sociales entre los individuos de uno y otro sexo y provoca la necesidad de replantear el concepto de historia.

Con esta orientación, encontramos artículos y libros cuyo objetivo es examinar la revolución desde otra óptica $y$ desde un marco conceptual que vincule a las mujeres, no sólo al contexto histórico nacional sino a problemas específicos de género capaces de resolver lo que significan los papeles que juegan en los distintos procesos sociales.

En esta corriente ubico los artículos acerca de Hermila Galindo ${ }^{50}$, Carmen Serdán ${ }^{51}$ y las antologías $E l$ álbum de la mujer. Antología ilustra-

mujeres en México desde ta conquista hasta la épocia actual, tanto en México como en listados Unidos, ponencias que presentaron dentro del Simposio de llistoriografia mexicanista que se llevó a cabo en Oaxtepec, Morelos en 1990. Véasc Memorias del Simposio de hisloriografia mexicanista, Comité Mexicano de Ciencias IIistóricas/111, México, 1990.

50 (jano, "listricta" en Memoria, 1991, pp. 16.3-171. Y también, "Foministas", en Debate feminis/a, año 2, vol. 4, scpticmbre 1991, pp. 269-292 y cl citado "Revolución, (1915-1910)", of) cil.

51 Ramos, "Carmen Scrdán", en fiem, año 13, núm. 74, febrero 1989, p. 25-27. 
da de las mexicanas, (El porfiriatoy la Revolución, 52 Mujeres y Revolución 1900-191753 y el de Esperanza Tuñón, ${ }^{54}$ recientemente aparecidas ya que presentan nuevas líneas de investigación $e$ interrogantes que permitirán avanzar en la comprensión de un movimiento social que todavía tiene mucha tela de donde cortar.

Para terminar, debo subrayar que el estudio e investigación sobre los antecedentes de las luchas de las mujeres, es una condición sine qua non porque no sólo descubre su vigencia en el presente sino que cuando se las vincula a las reivindicaciones permanentes que las militantes feministas han esgrimido y siguen demandando, adquieren importancia en tanto que nos las revelan como un grupo social actuante cuya condición de sujeto histórico no sólo es innegable, sino dinámico y actual.

\section{Biblografí}

Archivo Histórico de la Secretaría de la Defensa Nacional (AIISDN)

-Barrera Fuentes, Florencio, Crónicas y debates de las sesiones de la Soberana Convención revolucionaria, IN⿴囗н, Mćxico, 1965, 3 vols.

-Bremaunt\%, Alberto, El sufiagio femenino desde el punto de vista constitucional, Ediciones del Frente Socialista de Abogados, México, 1937.

52 Rocha (comp.) Álbum, op cit. y "Nucstras", en op. cit.

53 Lau y Ramos, Mujeres, op. cit.

54 Tuñón, Mujeres, 1935-1938, 1992.
-Cano, Gabriela, "En estricta justicia...un proyecto feminista en el movimiento constitucionalista" en Memoria del Congreso Internacional sobre la Revolución Mexicana, Gobierno de San Luis Potosi/Inehrm, 1991, pp. 163171.

-, "Revolución, feminismo y ciudadanía en México, (1915-1940) en Georges Duby y Michelle Perrot (dirs.), IIistoria de las mujeres, trad. Marco Aurelio Galmarini, 'Taurus Ediciones, Maclrid, 1993 , t. 5, pp. 685-695.

-, "Las feministas en Campaña. La primera mitad del siglo Xx", en Debate feminista, año 2, vol. 4, scpticmbre 1991, pp. 269-292.

- y Verena Radkau, Ganando espacios. IIstorias de vida: Guadalupe Zúniga, Alura Flores $y$ Josefina Vicens. 1920-1940, UAM-I, Mćxico, 1989, 138 pp. (Col. Correspondencia).

-Diario de los Debates del Congreso Constituyente, 1916-1917, t. II, Imprenta del Gobierno, México, 1917.

-Filosofica y Letras. Revista de la Facultad de liilosolia, enero-diciembre de 1956, t. $\mathrm{xxx}$, núms. 60-62.

-lisher, Lillian Estelle "The influence of the present Mexican Revolution upon the status of mexican women" en The IIispanic American IIistorical Revieu, vol. XXII, núm. 1, pp. 211-228.

-Gónzalez Navarro Moisés, "El Porliriato. Vicla social" en Daniel Cosio Villegas (coord.), Historia Moderna de México, Editorial llermes, México, 1957.

-Hernández, Ana María, La mujer mexicana en la industria textil, Tipogralía Moderna, México (Sría. del Trabajo y Previsión Social), 1940.

-Ibarra de Anda, F., El periodismo en México, (vol. II: Las mexicanas en el periodismo), Edilorial Juventa, México, 1937.

La Mujer modema, San Antonio, Tcxas, diciembre 1909, t. I, núm. 1. 
La mujer Moderna, Semanario ilustrado, año 1, núm. 1, septiembre 16, México, 1915.

La mujery el movimiento obrero mextcano en el siglo XIX. Antología de la prensa obrera, Censmo, México, 1975.

-Lagarde, Marcela, "Hacia una memoria feminista" en $E l$ Machete, septiembre de 1980.

-Lau, Ana y Carmen Ramos, (Introd. y comp.), Mujeres y Revolución 19001917, INEHRM/INAII, México, 1993.

-Macías, Anna, "The mexican Revolution was no Revolution for women" en Lewis Hanke, ed., Latin America, a historical reader, Little Brown, 1974.

__, "Women and the mexican Revolution, 1910-1920" en The Americas, julio de 1980, vol. 37, núm. 1, pp. 53-82.

-, Against all odds. The feminism movement in Mexico to 1940, Greenwood Press, Westport, Connecticut, 1982 .

, "Felipe Carrillo Puerto y la liberación de las mujeres en México" en Asunción Lavrin (comp.), Las mujeres Latinoamericanas. Perspectivas históricas, FCE, México, 1985, pp. 329-346.

-Martínez Vda. de Hernández, Aurora, Antorchas de la Revolución, Gráfìcos Galeza, México, 1964.

Memorias del Simposio de historiografia mexicanista, Comité Mexicano de Ciencias Históricas/11H, México, 1990.

-Mendieta Alatorre, Ángeles, Juana $B$. Gutiérrez de Mendoza, 1875-1842. Extraordinaria precursora de la Revolución mexicana, INEHRM, México, 1983.

-, Carmen Serdán, Centro de Estudios Históricos de Puebla, México, 1971.

- La mujer en la Revolución mexicana, INEHRM, México, 1961.

1916 El Primer Congreso Feminista de Yucatán. Anales de esa memorable Asamblea, INFONAVTT, México, 1975 (Edición facsimilar del Primer Congreso Femi- nista de Yucatán, Mérida, Edición Atenco Peninsular, 1916.)

-Ortega, Andrés "El feminismo. Discurso pronunciado por el Sr. Lic. D. Andrés Ortega en el acto de ser recibido como socio en la Sociedad Mexicana de Geografia y Estadística, el jueves 13 de junio de 1907 " en Boletín de la Soctedad Mextcana de Geografía y Estadística, 5a. época, t. II, 1907.

-Parcero, María de la Luz, Condiciones de la mujer durante el siglo XIX, INAII, México, 1992 (Col. Cientílica, 264).

-Pasternac, Nora, "El periodismo lemenino en el siglo XIX. Violetas del Anáhuac" en Ana Rosa Domenclla y Nora Pasternac (eds.), Las voces olvidadas. Antología critica de narradoras mexicanas nacidas en el siglo $X I X$, El Colegio de México/PIEM, México, 1991, pp. 399-448.

-Ramos, Carmen, "Carmen Serdán. Mujer de su tiempo", en Fem, año 13, núm. 74, febrero 1989 , pp. 25-27.

- (comp.), El género en perspectiva, de la dominación universal a la representación múltiple, UAM-I, México, 1991, pp. 231-278.

-Rascón, Ma. Antonieta "La mujer y la lucha social en la historia de México" en Imagen y realidad de la mujer, SepSetentas, México, 1975, pp. 139-174.

- Ríos Cárdenas, María, La mujer mexicana es ciudadana. Historia con füsionomía de una novela de costumbres (19301940), $\Lambda$. del Borque, México, 1942.

-Robles de Mendoza, Margarita, La evolución de la mujer en México, s.e., México, 1931.

-Rocha, Martha Eva "Nuestras propias voces. Las mujeres en la Revolución Mexicana", en Historias, Dirección de Estudios Históricos del INAIl, México, octubre 1990-marzo 1991, núm. 25, pp. 120-121.

- , Il Álbum de la mujer. $\Lambda n$ tología ilustrada de las mexicanas. El porfirialo y la Revolución, INAll, México, 1991, vol. IV (Col. Divulgación). 
-Rodríguez Cabo, Matilde, "La mujer y la Revolución", s.e., México, Conferencia dictada en el frente Socialista de Abogados, 1937.

-Sáenz Royo, Artemisa, Semblanzas: mujeres mexicanas, revolucionarias y guerreras, revolucionarias ideológicas, Manuel León Sánchez, México, 1960.

, Historia político social cultural del movimiento femenino en México, 1914-1950, Manuel León Sánchez, México, 1954.

-Soto, Shirlene Ann, "Yucatán's leadership in the women's movement: the first and second feminist congresses, 1916", California State University, Northridge, $1985,12 \mathrm{pp}$.

, The mexican woman: a study of her participalion in the Revolution. 1910-1940, California, Palo Alto, 1969.

-Tuñón, Esperanza, Mujeres que se organizan. El Frente Único Pro Derechos de la Mujer, 1935-1938, Miguel Ángel Porrúa/Coordinación de Humanidades, UNAM, México, 1992.

Turner, Frederick C. "Los efectos de la participación femenina en la revolución de 1910" en Historia Mexicana, vol. 16, núm. 4, abril-junio de 1967.

La dinámica del nactoralismo mexicano, Grijalbo, México, 1971.

-Urquizo, Francisco L., Recuerdo que... INEi IRM, México, 1985.

-Vallens, Vivian, Working uomen in Mexico during the Porfiriato, 18801910, R\&F Research Associates, San Firancisco, 1978.

-Vidales, Susana, "Ni madres abnegadas ni adelitas" en Crílicas de la economía política, lì Caballito, México, 1980, núm. $14 / 15$.

-Violetas del Anáhuac. Periódico literario, redaclado por señoras. Tipogralía de Aguilar e Ilijos, México, 1888.

-Zamora, IEmilio "Sara Istela Ramírez: una rosa roja en el movimiento" en Magdalena Mora y Adelaita R. del Castillo (cds.), Mexican uomen in the United States, struggles past and present, Chicano Studies Research Center Publications/University of California, Los Angeles, 1980, pp. 16.3-169. 